\title{
Development of Age Determination Technique Part 1: Neural-Network Based Prediction of Chum Salmon Age by Scale Images
}

\author{
Ryoma Hoson $^{1}$, Hiroyuki Shioya ${ }^{1}$, Yasuyuki Miyakoshi ${ }^{2}$, Fumi Yamaguchi ${ }^{3}$, and Hirokazu Urabe ${ }^{3}$ \\ ${ }^{1}$ Muroran Institute of Technology, 27-1 Mizumoto, Murorani, Hokkaido, Japan \\ ${ }^{2}$ Kitami Salmon Enhancement Program Association, 5-17 Shinmachi 1, Abashiri, Hokkaido, Japan \\ ${ }^{3}$ Hokkaido Research Organization, Fisheries Research Department, Salmon and Freshwater Fisheries Research \\ Institute, 3-373 Kitakashiwagi, Eniwa, Hokkaido, Japan
}

Keywords: salmon scale, age identification, automatic extraction, neural networks

Introduction

In recent years, the poor catch of chum salmon has continued in Japan, and the research aimed at monitoring the stock status and elucidating the factors that cause fluctuations in stock has been attracting attention. However, since it takes a lot of time to perform age assessment by hand, it is required to actively introduce information technology such as AI and ICT. Neural networks have been applied to predict or represent fisheries data, and many works in the field of fisheries have been presented (Hyun et al. 2005; Suryanarayana et al. 2008; Zhou 2003). In this study, we constructed an age-assessment system using neural networks to automate salmon age determination using scale images and verified its effectiveness. Salmon age determination was composed as the computational process by using neural networks with respect to the image classification. The age-assessment system will contribute to the analysis of salmon data in Hokkaido.

\section{Salmon scale and age determination}

Salmon scales have been corrected for use in the investigation of the age distribution of salmon returns. The data of 20,000 tails are summed in every year, and the scales are cleaned and transcribed onto a plastic plate as replica samples. The samples have been corrected for over 20 years. Three images of Fig. 1 present the press machine, a replica sample, and an extracted scale image, respectively. The enlarged images from the replica samples were used for age determination. The magnifying glass device provided a fine image of the salmon scale.
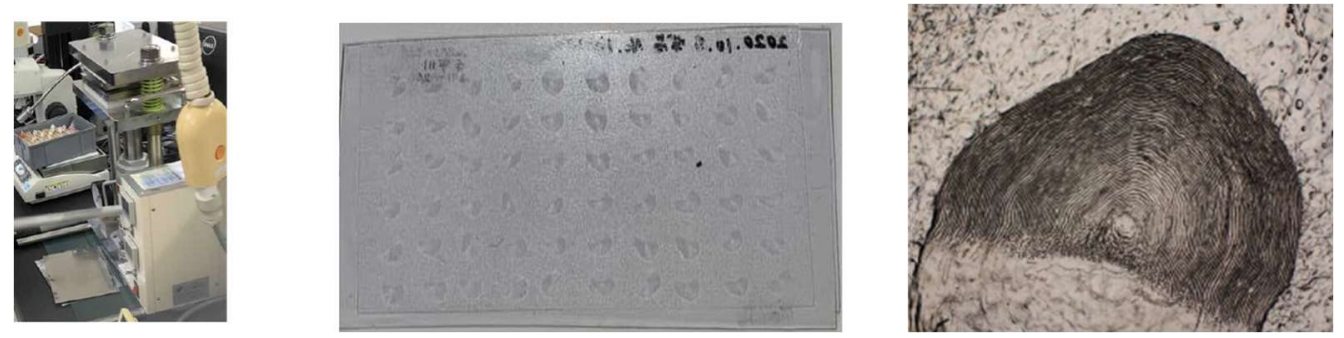

Fig. 1. Press machine (left), replica sample (middle), and microscope scale image (right).
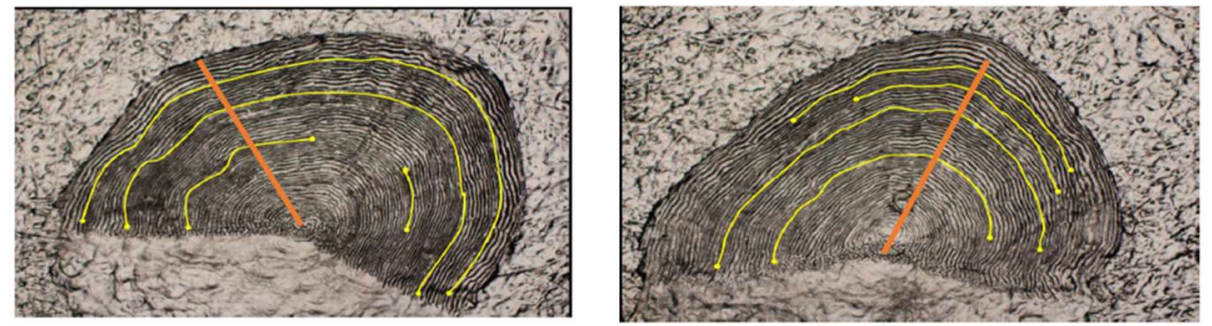

Fig. 2. Scale image of four years (left) and scale image of five years (right).

Circuli appear as curved and striped patterns arranged around the center of the scale. Each narrow pattern of circuli indicates a period of reduced scale growth each year. The age of a salmon is measured by counting the areas of narrow circuli, starting from the center of the scale and moving in a straight line to the edge. Each narrow pattern is considered one year of life, with the edge of the scale being considered a year as well. In Fig. 2, two scales are presented, and the left image is a scale with four years of growth, and the right image presents a scale with five years of growth. The yellow lines are the annuli determined by the expert Fisheries Institute staff. There are many types 
of narrow patterns, and assessment work, supported by Fisheries Institute staff, takes a long time. A scale image is divided into two parts: the narrow annuli and the other. Such the annuli indicate the period during each year.

Neural networks have been used for various classification problems. Multilayer-type networks have often been called the multilayered perceptions for constructing the recognition procedure. In this study, we used two neural networks to extract the center and annuli from the scale. The annuli data consists of the training data indicating the year-period marks. The Fig. 2 (right) presented the data processing, binary filter, segmentation, detection process from the center of the scale and the annuli. The outputs by the two neural networks are used for evaluating salmon age.

\section{Scale classification based on neural networks}

Neural networks have been used for image or pattern recognition, data learning, and classification problems. Multilayer-structured neural networks are useful for various data-learning problems in engineering and scientific fields. Here, we focused on a simple classification problem for the salmon scale, and then used multilayer neural networks to solve this problem. There are two classifications for age determination: one is the center of the scale, and the other is the annuli. We introduced two neural networks for the center and year period detection procedures. We set a Type-1 network used for detecting the center from cropped partial images. A Type- 2 network was for the annul according to the order of determination.

The structure of the Type- 1 network is the four-layer feed-forward type, and two hidden layers were used. The size of the cropped images from scale were $200 \times 68$ pixels. The number denoted the image size and the number of input layer nodes of the Type-1 network was 13,600. The number of nodes in the hidden-layers was 1,500. A sigmoid type activation function was used for the output node. The output layer consists of one node for classifying two states, center or not. The structure of the Type- 2 network was five layers of feed-forward neural net. Three hidden layers were used. The image size for classifying the annuli was $50 \times 34$ pixels. The number of input layer nodes of the Type-2 network was 1,700. The numbers of three hidden-layer nodes were 750, 1,500, and 750, respectively. The output layer with one node was used for classifying the annuli or not. The activation function of the output node was the same as the Type- 1 network. Figure 3 presents the procedure for the age determination process: the data processing, binary filter, segmentation, and detection process from the center of the scale and the annuli. The outputs by the two neural networks are used for evaluating salmon age.
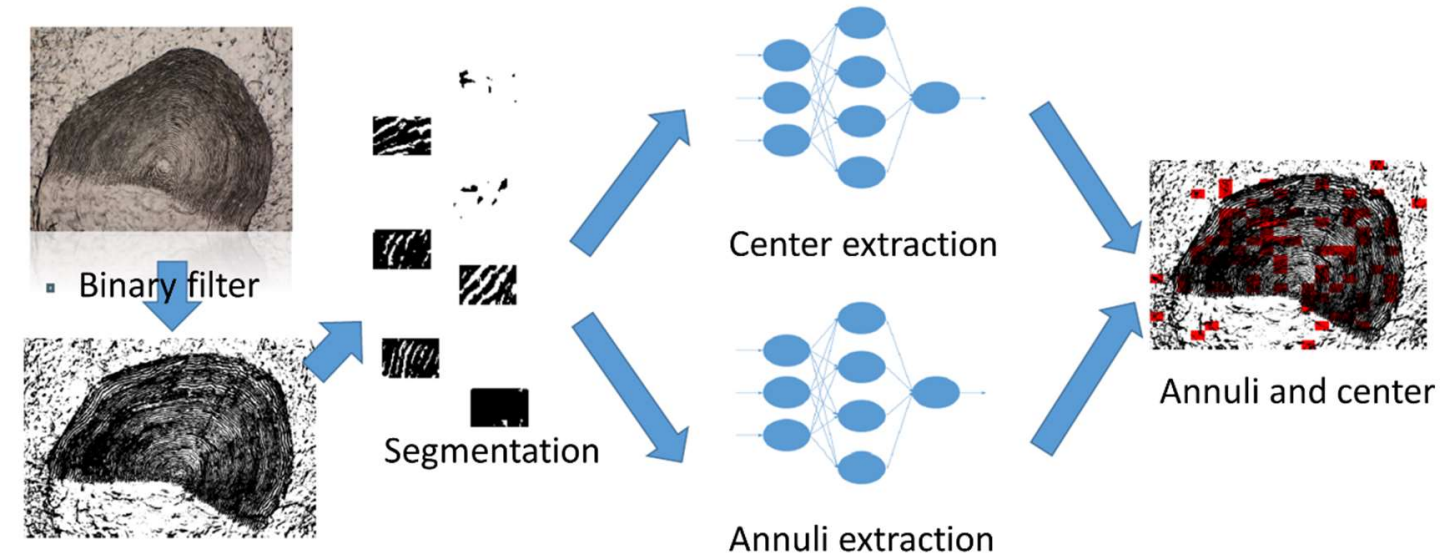

Annuli extraction

Fig. 3. Schematic figure of the computational procedure using neural networks for extracting the center and annuli.

The computational procedure for both neural networks was composed of Python 3.6 and Keras software. As concerning the optimizer for the data training procedure, we used Adam with a certain settled parameter set. About 500 iterations for the training process were required for Type- 1 neural network. However, about 2000 iterations of the data training were required in Type-2 neural network. The performance for the untrained data set for the overtrained neural network is inferior to the optimal-trained network. We settled the training iterations for obtaining the optimal trained neural nets. The centers and annuli were obtained by the networks. The center and the arrangement of year periods were used as the count of each year. The annuli as the year-period marks were classified by a criterion using the nearest distance in the direction through the center. 


\section{Computational results and summary}

The scale image with the year-period marks and center is presented in Fig 2. The yellow lines indicate the year-period marks by the fishery Institute staff. The detected marks by the neural network almost conformed to the lines. The accuracy ratios of the annuli for training and test data were $73.6 \%$ and $71.7 \%$, respectively. In this study, we used only 60 tails of salmon obtained recently. Then, the reliability of the neural network should be developed using many samples. The stability in training was indicated by almost the same performance for the test and training data. The age-determination system with two neural networks provided the annuli and center from the salmon scale, and the system is helpful to realize the automatic age-assessment for the salmon returns in Hokkaido.

\section{REFERENCES}

Hyun, K., M.Y. Song, S. Kim, and T.S. Chon. 2005. Using an artificial neural network to patronize long-term fisheries data from South Korea. Aquat. Sci. 67(3): 382-389.

Suryanarayana, I., A. Braibanti, R.S. Rao, V.A. Ramamc, D. Sudarsan, and G.N. Rao. 2008. Neural networks in fisheries research. Fish. Res. 92(2-3): 115-139.

Zhou, S. 2003. Application of artificial neural networks for forecasting salmon escapement. N. Am. J. Fish. Manag. 23(1): 48-59. 